\title{
Characterization and estimation of refractive index profile of laser-written photopolymer optical waveguides
}

\author{
Mehmet Salih Dinleyici* , Can Sümer \\ Electrical and Electronics Engineering, Izmir Institute of Technology, Gulbahce-Urla, 35430, Izmir, Turkey
}

\section{A R T I C L E I N F O}

\section{Article history:}

Received 4 April 2011

Received in revised form 31 May 2011

Accepted 11 July 2011

Available online 29 July 2011

\section{Keywords:}

Photopolymer optical waveguides

Refractive index modulation profile

Fresnel-Kirchhoff diffraction

Direct-writing

\begin{abstract}
A B S T R A C T
In this study, channel waveguides fabricated in photopolymer films by direct-writing using a low-power CW laser, are used as phase objects in a simple plane-wave diffraction setup, and the refractive index modulation profiles of the waveguides are characterized using the recorded diffraction patterns. Index profiles are modeled by piece-wisely combining two Gaussian functions representing the central and the tail regions. Measured diffraction patterns are matched with patterns generated using the model. This simple model makes it possible to design various channel waveguides embedded into polymer substrates. The proposed model is tested on three distinctive waveguide profiles written on the same Acrylamide/Polyvinyl Alcohol based photopolymer with different exposures.
\end{abstract}

(C) 2011 Elsevier B.V. All rights reserved.

\section{Introduction}

Photonic planar lightwave circuits have been attracting significant attention in the field of all-optical communication. Complex functions of optical communication devices require compound multifunction components assembled on a single photonic substrate. Because of the relative ease in the realization of functional devices, polymeric films have become promising candidates for materials used in the fabrication of such devices. There are many proposed devices based on polymer structures which function as coupling, switching, and filter elements [1-3]. All these devices are essentially composed of channel waveguides. The fabrication of these waveguides traditionally utilizes photolithographic techniques or reactive ion etching. Such processes are expensive, time consuming and impractical. Alternatively, non-lithographic fabrication using laser direct-writing techniques have also been proposed [4,5]. Main difficulties in this technique are the requirement of high laser power and difficult precision alignment. Recently, azobenzene dye-functionalized polymer films have been successfully demonstrated for laser directwriting fabrication of stable polymer channel waveguides, using a $5 \mathrm{~mW}$ CW DPSS laser operating at $532 \mathrm{~nm}$ [6].

In all direct-write optical devices, effective refractive index profile of the channel waveguide is an important parameter for designing highly efficient, low loss structures. Currently, prism coupling technique has been widely used with WKB method for the estimation of the profile $[7,8]$. This method is based on the propagating modes of the waveguide,

\footnotetext{
* Corresponding author. Tel.: + 90 2327506575; fax: +90 2327506599. E-mail address: salihdinleyici@iyte.edu.tr (M.S. Dinleyici).
}

which do not allow local measurement of the waveguide cross-section. Furthermore, it requires a relatively complex setup and time-consuming numerical computations for effective profile estimation.

In this paper, we propose a diffraction method based on FresnelKirchhoff diffraction integral under paraxial approximation, and smooth change of the refractive index profile. The approach was applied for estimating the optical fiber core refractive index and diameter [9]. Here, the model is adapted for a channel waveguide geometry which is laser direct-written in photopolymer substrates. It is applied for various channel waveguides which were formed by multiple-pass laser directwriting and has proven to be very sensitive in refractive index changes in the order of $10^{-5}$. For an Acrylamide/Polyvinyl Alcohol based photopolymer dry film, a refractive index modulation of up to $2.8 \times 10^{-3}$ was reported in [10], yet the peak modulation is estimated to be $1.3 \times 10^{-3}$ using a $0.5 \mathrm{~mW}$ CW laser in this study.

\section{Mathematical model}

The proposed mathematical method is based on Fresnel-Kirchhoff diffraction theory. This method, modified for the phase objects under paraxial approximation, was recently applied to estimation of the optical fiber core refractive index in [9]. In brief, when a plane wave propagates through the phase object its phase front is proportionately modified along the polymer film (phase object) and diffraction occurs between the exit surface of the film and an observation plane. Following this idea, the mathematical method is applied to the estimation of the refractive index profile of direct-written photopolymer optical waveguides which are considered as phase objects. The diffraction formulation is designated as one dimensional because the longitudinal profile is assumed to be constant along the waveguide. 

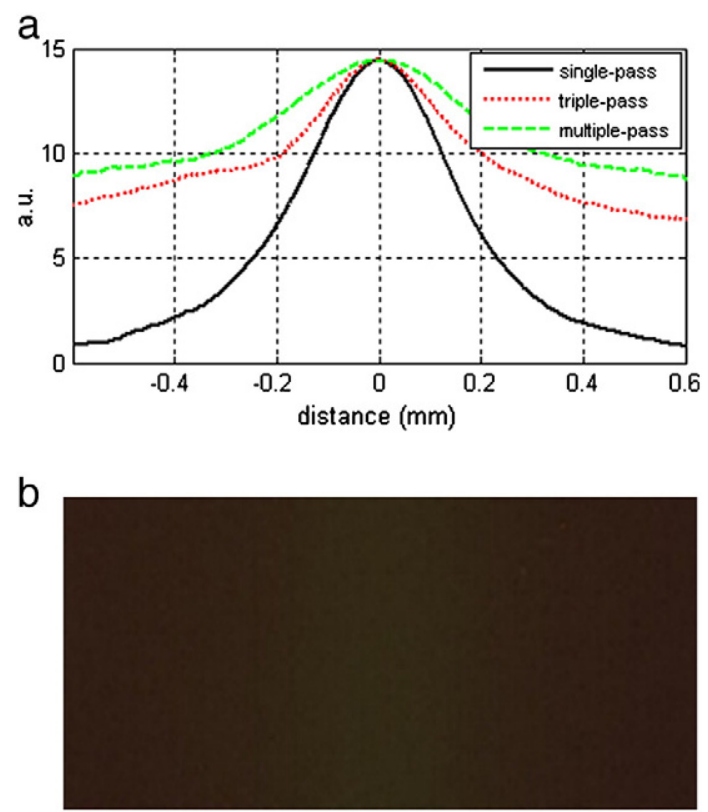

Fig. 2.1. a) Cumulative refractive index profiles and b) Picture of a channel waveguide.

A picture taken by a transmission optical microscope and the refractive index profiles of the waveguides to be estimated are shown in Fig. 2.1.

The index profiles are obtained by cumulative transmission of incoherent light (incandescent microscope light) through the film, not a true refractive index profile of the waveguide cross-section. Furthermore, depending on the writing procedure (exposure, laser beam profile) and the material diffusion properties (nonlocal diffusion of photopolymerization) the index profiles are expected to be Gaussian-type. Therefore, the profile can be approximated with a Gaussian function in the central region and another one in the tail region. In the direction of the propagation (depth) the profile can also be modeled with a Gaussian function as explained in [6]. In this paper, three different channel waveguides written on the same PVA photopolymer with different exposures are approximated and the Gaussian parameters are estimated. By taking the waveguide as a phase object between planes $\sum_{1}$ and $\sum_{2}$, the diffraction geometry is given in Fig. 2.2.

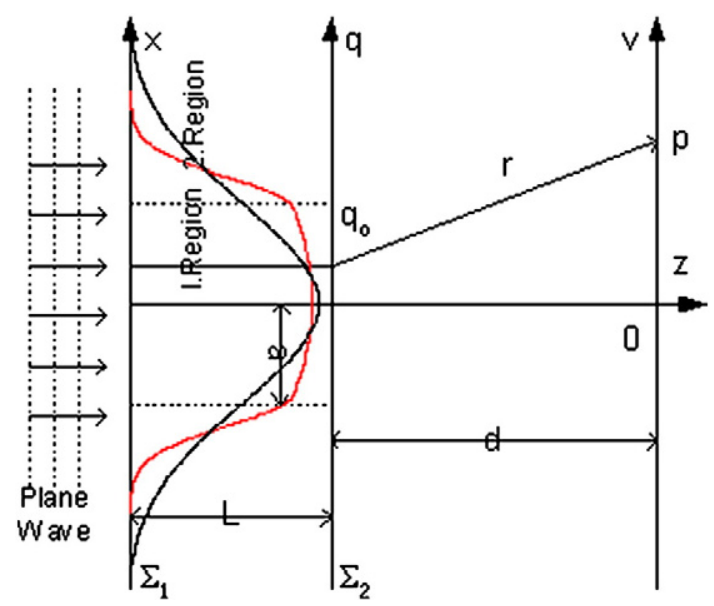

Fig. 2.2. Diffraction geometry.
The diffraction integral for the complex amplitude of the field at point ' $p$ ' on the observation plane is given in [9]:

$U(p)=\int \frac{A(q) \Gamma(q, p)}{r} \exp (i k r) d q$

where $A(q)$ is complex amplitude at the $\sum_{2}$ plane, which is the accumulation of the phase retardation through the index region, $k$ is the wavevector. $\Gamma(\mathrm{q}, \mathrm{p})$ is the inclination factor, because of faint and smooth index modulation, the inclination factor has insignificant effect on the diffraction pattern and assumed unity as discussed in the Appendix A in Section 6 . The intensity at point $p$ is given by $I(p)=U(p) U^{*}(p)$. The distance $\mathbf{r}$ between a point on plane $\sum_{2}$ and the observation point $p$ is approximated by $r(p) \approx d+(p-q) / 2 d$. In order to match the geometry of the index profile $\Delta \mathrm{n}(\mathrm{x})$, it is divided into two regions on each side. In the each region, the refractive index profile is modeled by piece-wisely combining Gaussian functions as depicted in Fig. 2.2.

The diffraction integral for the first region $-\mathrm{a} \leq \mathrm{q} \leq \mathrm{a}$ becomes,

$U_{1}(p)=K_{1} \int_{-a}^{a} \exp i\left\{\Delta n k T_{F} \exp \left(\frac{-x^{2}}{\beta^{2}}\right)+\frac{\pi}{\lambda d}(q-p)^{2}\right\} d q$.

For the second region $\mathrm{q} \geq \mathrm{a}$,

$U_{2}(p)=K_{2} \int_{a}^{\infty} \exp i\left\{\Delta n k T_{F} \exp \left(\frac{-(x-a)^{2}}{\sigma^{2}}\right) \exp \left(\frac{-a^{2}}{\beta^{2}}\right)+\frac{\pi}{\lambda d}(q-p)^{2}\right\} d q$.

Similarly for the negative side $\mathrm{q} \leq-\mathrm{a}$,

$U_{3}(p)=K_{3} \int_{-\infty}^{-a} \exp i\left\{\Delta n k T_{F} \exp \left(\frac{-(x+a)^{2}}{\sigma^{2}}\right) \exp \left(\frac{-a^{2}}{\beta^{2}}\right)+\frac{\pi}{\lambda d}(q-p)^{2}\right\} d q$

Then the total field and intensity at point $\mathrm{p}$ are given by

$\mathrm{U}(\mathrm{p})=\mathrm{U}_{1}(\mathrm{p})+\mathrm{U}_{2}(\mathrm{p})+\mathrm{U}_{3}(\mathrm{p})$ and $I_{p}=\left|U_{p}\right|^{2}$.

$\Delta \mathrm{n}(\mathrm{x}) \mathrm{k} \mathrm{T}_{\mathrm{F}}$ is the accumulative phase retardation due to propagation through the film at position $\mathrm{x}$, as shown in the Fig. 2.3.

a

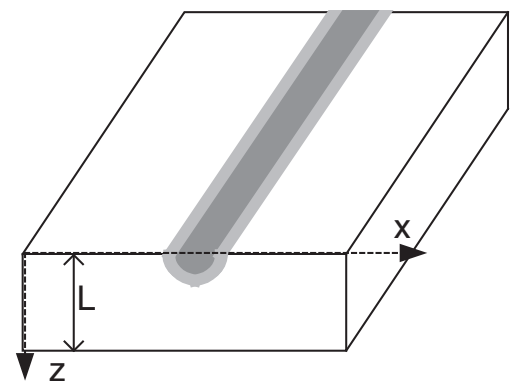

b

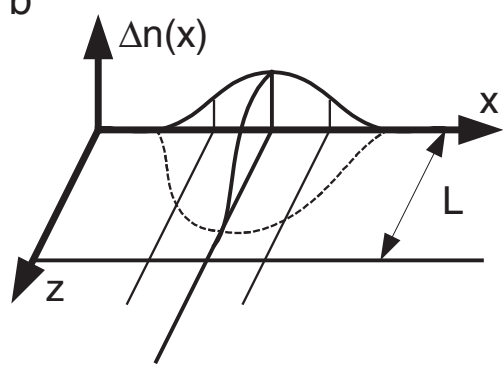

Fig. 2.3. a) Channel waveguide embedded into the film. b) Refractive index profile. 
a

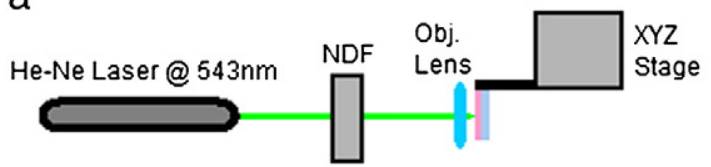

b

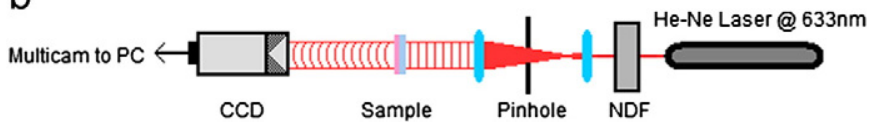

Fig. 3.1. Experimental setup: a) laser-writing and b) diffraction recording.

Assuming the index modulation having Gaussian exponential decreasing tendency and the inclination factor has insignificant effect, total phase retardation may be obtained by a line integral as follows,

$T_{F}=\int_{0}^{L} \exp \left(\frac{-z^{2}}{D^{2}}\right) d z \Rightarrow D \frac{\sqrt{\pi}}{2} \operatorname{erf}(L / D)$.

where $\mathrm{L}$ is the film thickness and $\mathrm{D}$ is the waveguide depth parameter. The integral results in a constant value $\left(T_{F}\right)$ as expected. Total diffraction intensity distribution can be obtained by applying the procedure for every point on the observation plane.

\section{Experimental procedure}

Photopolymer composition employed in waveguide forming is based on Acrylamide (AA) as the active material prepared in Polyvinyl Alcohol (PVA) matrix. Polymer recipe includes AA (Aldrich, $\mathrm{MW}=71.08)$ as the photoactive monomer, Yellowish Eosin (YE)

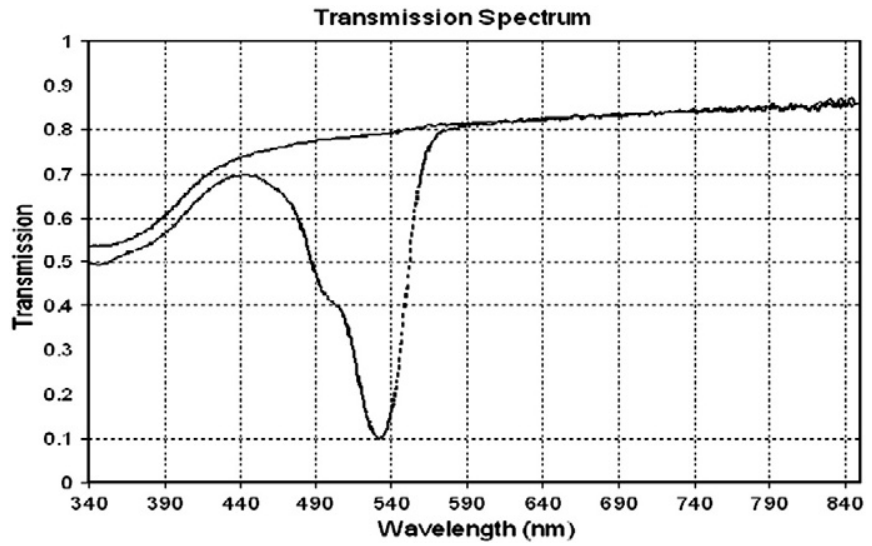

Fig. 3.2. Polymer transmission spectra for unprocessed (dashed) and polymerized (solid) samples.
(Sigma-Aldrich, MW $=647.89$ ) as photoinitiator, Triethanolamine (TEA) (Sigma-Aldrich, MW =149.19) as coinitiator, mixed in aqueous PVA (Sigma-Aldrich, MW $=30.000-70.000$ ) solution. The solution is prepared by adding $2.9675 \% \mathrm{w} / \mathrm{v}$ TEA, $3.17 \% \mathrm{w} / \mathrm{v}$ AA and $0.016175 \%$ $\mathrm{w} / \mathrm{v}$ YE into $10 \% \mathrm{w} / \mathrm{v}$ aqueous solution of PVA. Prepared solution is coated on microscope glass slide substrates by direct casting, and then the films are left for $24 \mathrm{~h}$ in the dark at room temperature to allow for the completion of PVA cure.

Channel waveguides are formed by direct-writing in photopolymer films using a $0.5 \mathrm{~mW}$ CW output from $543 \mathrm{~nm}$ He-Ne laser shown in Fig. 3.1.

Laser output is focused on the film using a $40 \times$ objective lens which makes $125 \mu \mathrm{m}$ beam diameter. The position and velocity of the focused beam are controlled using a computer-controlled 3-axis micropositioning stage. The photopolymer used in this paper has an absorption peak around $535 \mathrm{~nm}$ and the spectral characteristics before and after polymerization are shown in Fig. 3.2.

In order to observe diffraction of an incident plane wave from the waveguide, a plane-wave diffraction setup employing a He-Ne laser working at $633 \mathrm{~nm}$ is used and shown in Fig. 3.1. The pictures are recorded by a CCD camera having $720 \times 640$ pixels image sensor with $11 \times 11 \mu \mathrm{m}^{2}$ pixel area.

\section{Results}

Three different channel waveguides are written by the procedure explained in the previous section, a transmission optical microscope picture and their index profiles are shown in the Fig. 2.1. Therefore, the mathematical model proposed in this paper is based on the Gaussian functions in the lateral as well as in the depth direction.

The diffraction patterns for the three waveguides are recorded at $29.5 \mathrm{~mm}$ distance are shown in Fig. 4.1, where the CCD image and the corresponding cross-section profiles are given.

The material recipe is the same for all three-cases, but the laser exposure dosages are different. Waveguides obtained by the singlepass, triple-pass and multiple-pass direct writing are shown on the left, on the right and in the middle, respectively in Fig. 4.1. The velocity of the writing beam on the film surface is set as $0.5 \mathrm{~mm} / \mathrm{s}$. Hence, triple-pass corresponds to three times the exposure dosage of the single-pass, which means three times scanning. Similarly multiplepass is an exposure dosage of 10 times the single-pass.

In order to verify the procedure, the diffraction patterns of these three waveguides are compared with the estimated mathematical reconstructions. During the procedure, peak index modulation, observation distance and computational parameters are held constant. The index profile function parameters for the each region $(\beta, \sigma)$ and the depth (D) are adjusted for reconstruction of these three waveguides.

The single-pass waveguide diffraction and reconstruction curves are shown in Fig. 4.2, $\beta$ is $0.13 \mathrm{~mm}, \sigma$ is $0.055 \mathrm{~mm}$ and the depth $\mathrm{D}$ is

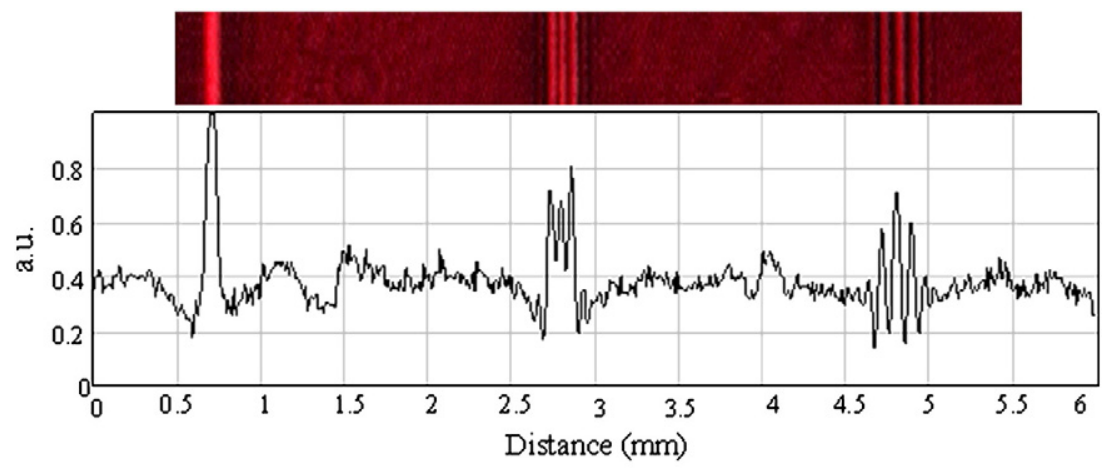

Fig. 4.1. Diffraction images and cross-section profiles. 


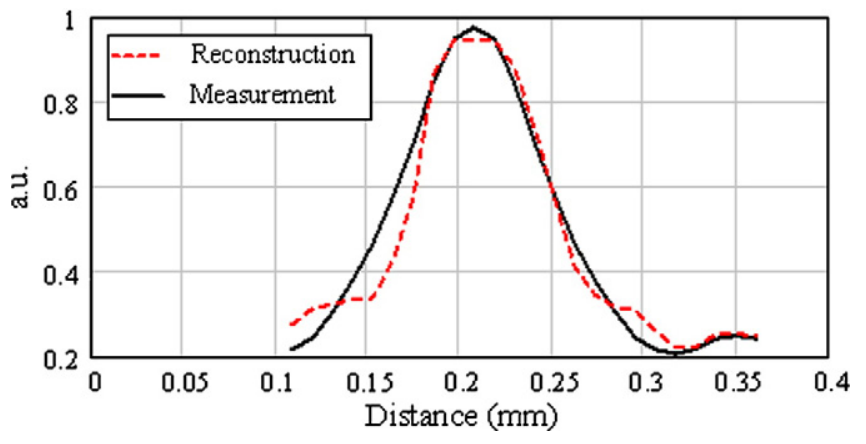

Fig. 4.2. Single-pass waveguide diffraction and reconstruction.

$0.25 \mathrm{~mm}$. In the figure, a half-pixel mismatch in the later direction and saturation at the center are apparent.

Triple-pass waveguide diffraction and reconstruction curves are shown in Fig. 4.3; $\beta$ is $0.11 \mathrm{~mm}, \sigma$ is $0.02 \mathrm{~mm}$ and the depth $D$ is $0.56 \mathrm{~mm}$. Because of the paraxial approximation and the camera noise floor, the reconstructed curve is inaccurate at the higher-order diffractions. Sensitivity of the procedure for peak index modulation may be obtained from this figure, as depicted for $5 \times 10^{-5}$ peak index change; peak intensity of the first-order diffraction peak reveals this information. Correspondingly, for holographic grating methods, the first-order diffraction peak is generally considered for characterization as in [10].

The final plot for the multiple-pass waveguide structure is shown with the reconstruction curve in Fig. 4.4 ; $\beta$ is $0.7 \mathrm{~mm}, \sigma$ is $0.075 \mathrm{~mm}$ and depth (D) is $0.4 \mathrm{~mm}$. Here, in the central region these two curves match with a good agreement. Only slight mismatch between two symmetric first-orders in the measured pattern must be due to measurement error (CCD pixel) since everything is symmetric in the procedure.

Obviously for the multiple-pass direct-writing waveguides the depth is increased and the profile widens in the central region; as a result the profile starts to deviate from the Gaussian profile. At higher angles the paraxial approximation starts to fail, and the curves follow the trends but do not match exactly. This may be due partly to integral convergence, since the integral computation parameters are kept constant throughout all reconstruction. Moreover, background stray lights make the measured intensity floor higher than it should be.

\section{Conclusion}

Laser direct-written photopolymer waveguides have diverse refractive index modulation profiles that depend on recipes and writing procedures even for the same photopolymer recipe.

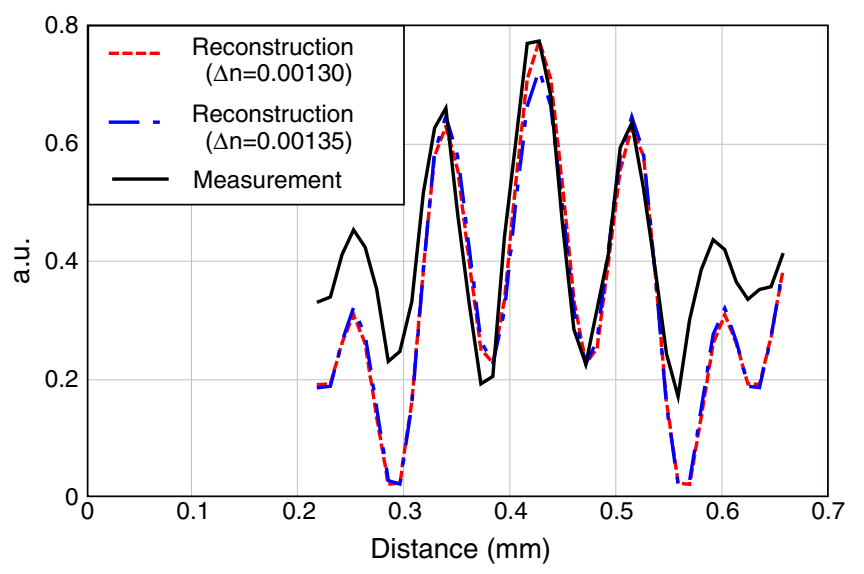

Fig. 4.3. Triple-pass waveguide diffraction and reconstruction.

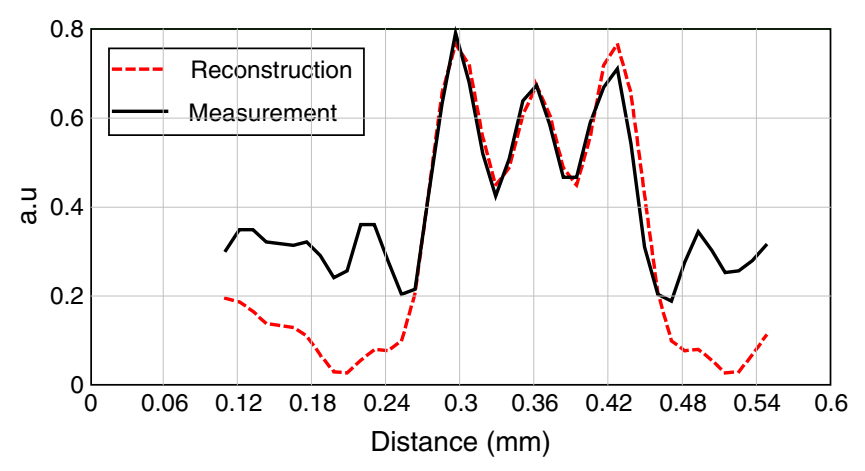

Fig. 4.4. Multiple-pass waveguide diffraction and reconstruction.

Therefore, a practical method is desirable for the profile estimation in order to design photonic integrated waveguide devices that function consistently.

Channel waveguides are fabricated via laser direct-writing on photopolymer films manufactured on glass slides. The films are prepared by direct casting of aqueous solution of Polyvinyl Alcohol polymer matrix encasing photopolymerizable Acrylamide monomer and respective catalysts. The aqueous solution is prepared by mixing weighed amounts of polymer constituents. Direct casting of polymer films is followed by a 24-hour PVA cure. For this recipe the peak index modulation was reported to be 0.0028 in [10]; however, in our case this is 0.0013 . This discrepancy may be due to laser wavelengths that differ a little (in our case $542 \mathrm{~nm}$ instead of $532 \mathrm{~nm}$ ) and also the laser powers are not the same.

The profile of the index modulation can effectively be reconstructed with Gaussian functions in lateral and depth directions. In this study, three distinctive direct-write channel waveguides are obtained by exposures with multiple (one, three and ten times) passes with a scan speed of $0.5 \mathrm{~mm} / \mathrm{s}$ and the profiles are approximated based on the Fresnel diffraction model. Although the diffraction patterns of the waveguides match with the models in the central parts, they begin to deviate at higher diffraction angles. This is partly due to integral convergence because the computational parameters are held constant for the three waveguides. Besides, the diffraction formulation provides better results under paraxial approximation. The refractive index sensitivity of the method is as high as $5 \times 10^{-5}$ which is suitable for design purposes. Therefore, the method can be employed for designing channel waveguide structures in polymer integrated photonic structures which frequently require evanescent optical signal coupling between waveguides or optical components. In such couplers the evanescent field is strongly affected by the waveguide profile and hence the coupling coefficient is altered. Also, in the design of direct-write diffraction gratings, channel waveguide index profiles are considerable factors for the diffraction efficiency.

Consequently, the proposed method can be employed wherever the local refractive index profile of channel waveguide is to be practically estimated for designing a photopolymer based lightwave circuit.

\section{Acknowledgment}

The authors are grateful to Dr. Mustafa M. Demir from Izmir Institute of Technology, Department of Chemistry for his assistance and facilities provided throughout this study. This work was supported in part by TUBITAK under Project 109E204.

\section{Appendix A}

The inclination factor under paraxial approximation and weak index modulation can be analytically proved to be insignificant for thin 
polymer films as follows. The refractive index modulation function is assumed as,

$$
\Delta n(x, z)=n_{o} \exp \left(-x^{2} / \beta^{2}\right) \exp \left(-z^{2} / D^{2}\right) .
$$

Using ray equation under paraxial approximation,

$\frac{d}{d z}\left(n(x, z) \frac{d x}{d z}\right)=\frac{\partial n(x, z)}{\partial x}$

$\frac{d^{2} x}{d z^{2}}-\frac{2}{D^{2}} \frac{d x}{d z}+\frac{2 x}{\beta^{2}}=0$

The solution of this Hermite differential equation is complicated, but simplifies when the index modulation in the direction of propagation is neglected.

$x(z)=C_{0} \exp \left(R_{1} z\right)+C_{1} \exp \left(R_{2} z\right)$
$R_{1,2}= \pm j \sqrt{2 \beta^{-2}}$

Initial conditions are,

$$
\begin{aligned}
& x(0)=X_{0} \\
& \frac{d x(z)}{d z}=0
\end{aligned} \quad z=0 .
$$

The inclination angle is taken zero at $\mathrm{z}=0$ at the film surface $\left(\sum_{1}\right)$ because of the plane wave approximation for the incident wave.

$x(z)=X_{0} \cos \left(\frac{\sqrt{2}}{\beta} z\right)$

Then, $\Gamma(\mathrm{L})$ : inclination angle at the film exit surface $\left(\sum_{2}\right)$ is given by

$\Gamma(L)=\frac{d x(z)}{d z}$ at $\mathrm{z}=\mathrm{L}$.

There are two important points for the rays arriving at $\sum_{2}$ plane. First the angle of inclination and second the displacement from the originating point. Eq. (A.6) gives the displacement for the ray originating

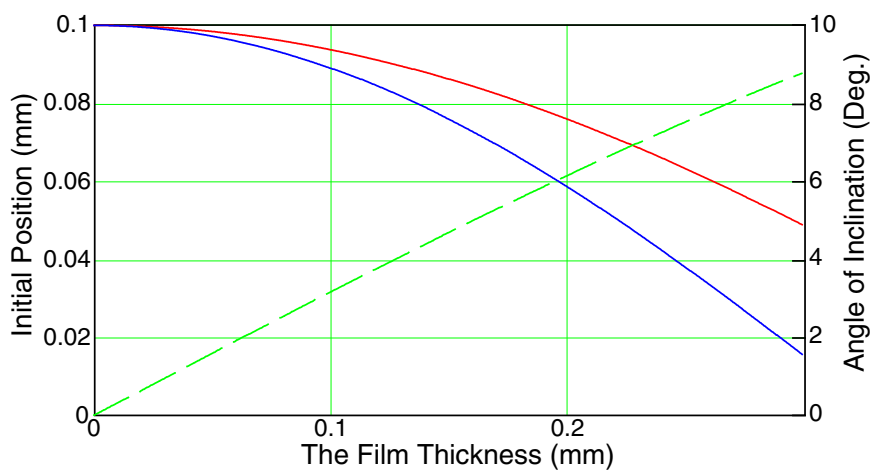

Fig. A.1. Inclination angle and Ray displacement.

from $\mathrm{X}_{0}$, while Eq. (A.7) is for the inclination angle. Fig. A.1 shows the displacement of the rays from the initial position for two different $\beta$ values. The deviation depends strongly on the film thickness and $\beta$, but the mapping is one-to-one for the rays for the given $\beta$. Therefore, the diffraction pattern will not be adversely affected but scaled up or down.

This characteristic may be explained with the convergence properties of exponential index modulation profile generated in the polymer film. For the given film thickness, the inclination angle is only a few degrees at the film exit surface as shown in the Fig. A.1 for rays originating from $0.1 \mathrm{~mm}$ initial position, which has negligible effect on the diffraction integral. Therefore, the inclination factor can be assumed to be a constant for evaluation of the diffraction integral.

\section{References}

[1] H. Ma, A.K.Y. Jen, L.R. Dalton, Adv. Mater. 14 (2002) 1339.

[2] Y.-C. Jeong, S. Lee, J.-K. Park, Opt. Exp. 15 (2007) 1497.

[3] N. Keil, H.H. Yao, C. Zawadzki, F. Beyer, O. Radmer, M. Bauer, C. Dreyer, Electron. Lett. 41 (2005) 186

[4] R. Sigel, G. Fytas, N. Vainos, S. Pispas, N. Hadjichristidis, Sci. 297 (2002) 67.

[5] N. Srisanit, Z. Liu, X. Ke, M.R. Wang, Opt. Commun. 244 (2005) 171.

[6] Z. Liu, N. Srisanit, X. Ke, P. Wu, S. Song, J.J. Yang, M.R. Wang, Opt. Commun. 273 (2007) 94.

[7] P.K. Tien, R. Ulrich, R.J. Martin, Appl. Phys. Lett. 14 (1969) 291

[8] S. Schutzmann, M. Casalboni, F.D. Matteis, P. Prosposito, J.Non-Cryst.Solids 351 (2005) 1814.

[9] A. Sabatyan, M.T. Tavassoly, Opt. Eng. 46 (2007) 128001-1.

[10] C. Garcia, I. Pascual, A. Costela, I. Garcia-Moreno, C. Gomez, A. Fimia, R. Sastre, Appl. Opt. 41 (2002) 2613. 